\title{
Altitude Control Feasibility for a Seaweed Harvester
}

\author{
Marco Gallieri \\ Dipartimento di Ingegneria, Informatica, Gestionale e dell'Automazione \\ Universita' Politecnica delle Marche \\ Ancona, Italy \\ Email: gallieri.marco@gmail.com
}

\author{
John Ringwood \\ Department of Electronic Engineering \\ National University of Ireland \\ Maynooth, Co. Kildare, Ireland \\ Email: john.ringwood@eeng.nuim.ie
}

\begin{abstract}
In this paper, the feasibility of the altitude control of a seaweed harvester is examined. The harvesting system consists of a vessel and a suspended harvester device, the altitude of which is controlled by a winch. The goal of the control action is to maintain the harvester at a constant altitude with respect to the seabed profile. A control strategy is proposed, including a vessel motion feed-forward action, using a motion reference unit (MRU), and an altitude feedback loop, using a sonar device for altitude measurement.
\end{abstract}

\section{INTRODUCTION}

The seaweed harvester system under consideration is shown in Figure 1. The challenge of maintaining a suspended device at a specific distance from an undulating sea bed is new, though the solution we adopt draws upon considerable prior art in the area of marine systems and hydrodynamics. In particular, the body of knowledge available on offshore cranes is particularly relevant [1]-[3]. We develop a detailed hydrodynamic model for the vessel/harvester system, which relates the heave variations in the harvester to the wave motions experienced by the vessel, upon which model-based control designs can be built. In particular, we are interested to see if it is feasible to control the harvester altitude to within $\pm 10 \mathrm{~cm}$. This is necessary in order to maintain an optimum cutting height while avoiding any environmental damage to the seabed. The ship is subject to ocean wave disturbances, and moves at an average constant speed, with a periodical turning manoeuvre, in relatively shallow water.

The feed-forward control strategy proposed requires a onestep prediction and several prediction strategies are formulated, and compared. A gain scheduled PID controller is also formulated, and used for baseline comparison. A simulator, obtained by modifying the Fossen et al [4] Simulink MSS Toolbox, is used to evaluate the control performances, and the control results of the two control strategies are compared and discussed.

The paper addresses the problem as follows: Section II introduces the hydrodynamic reference frames which will form the setting for the hydrodynamic model for the vessel/harvester system. The dynamics of the vessel and the winch system are detailed in Section III, along with any simplifying modelling assumptions. Section IV details both the gain-scheduled PID and feedforward/feedback control designs, the tracking response of which are documented in Section V. Finally, conclusions are drawn in Section VI.

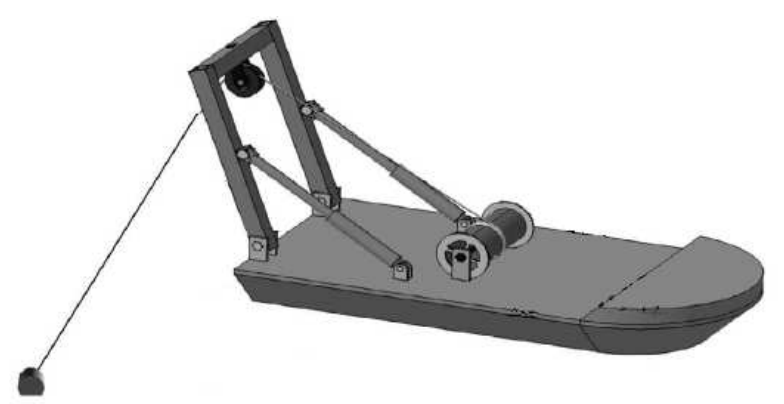

Fig. 1. The seaweed harvester [1]

\section{REFERENCE FRAMES}

The ship and harvester model, presented here will have 9 degree of freedom (DOF), and its coordinate systems are shown in Figure 2. For the vessel subsystem, the following right-hand reference frames are considered, as in [5]:

- North-east-down frame ( $n$-frame).

The $n$-frame, $\left(o_{n}, x_{n}, y_{n}, z_{n}\right)$, is used to define the absolute position of the vessel. The $x$-axis points towards the north, the $y$-axis towards the east, and the $z$-axis towards the center of the Earth.

- Ship body-fixed frame ( $b_{1}$-frame).

The $b_{1}$-frame, $\left(o_{b_{1}}, x_{b_{1}}, y_{b_{1}}, z_{b_{1}}\right)$, fixed to the hull, is used to formulate the equations of motion. The $x$-axis points towards the direction of motion, the $y$-axis towards starboard, and the $z$-axis points downwards. Together with the $n$-frame, the $b_{1}$-frame also defines the orientation of the vessel. For marine vehicles, the axes are chosen to coincide with the principal axes of inertia [5].

- Hydrodynamic ship frame ( $h_{1}$-frame)

The $h_{1}$-frame is used to compute the force and the motion due to interactions between the hull and the waves. The frame, $\left(o_{h_{1}}, x_{h_{1}}, y_{h_{1}}, z_{h_{1}}\right)$, is not fixed to the hull, and moves with the constant average speed of the vessel, following its path. Therefore, the wave-induced forces, computed in this inertial reference frame, make the vessel oscillate with respect to the $h_{1}$-frame.

For the harvester motion, the following right-hand reference frames are considered:

- Hydrodynamic harvester frame ( $h_{2}$-frame).

The $h_{2}$-frame, $\left(o_{h_{2}}, x_{h_{2}}, y_{h},, z_{h},\right)$, is not fixed to the harvester, and is used to compute the force due to interactions between the harvester and the waves. As 


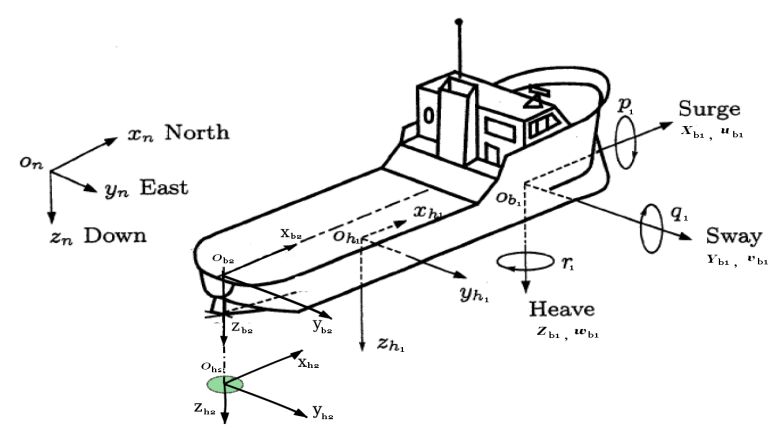

Fig. 2. Reference frames

for the $h_{1}$-frame, the $h_{2}$-frame moves at the average speed of the vessel, following its path. This reference frame is considered to be inertial, and the frame origin is determined such that the $z$-axis passes through the timeaveraged position of the harvester center of gravity.

- Winch body fixed frame ( $b_{2}$-frame).

The $b_{2}$-frame is fixed to the winch, and its origin, $\left(o_{b_{2}}, x_{b_{2}}, y_{b_{2}}, z_{b_{2}}\right)$, coincides with the center of the winch motor axis. The rope is assumed to be a rigid element, without mass, therefore the harvester and the rope are considered to be a single rigid body, and the winch $b_{2}$ frame is used for computing the harvester equations of motion and the motor equations.

\section{VESSEL AND WINCH DYNAMICS}

The 9 DOF dynamical model, detailed in this section, includes the effects of a forward vessel speed, the wave loads, the ship propellers and motion control forces, and the winch motor dynamics. This formulation arises from the unified ship model, given in [5], [6] and includes the seakeeping fluid memory effects. From a control prospective, the design becomes more challenging, because the wave induced disturbance is considered to be acting on the system input, rather than the output. The force response amplitude operator (Force-RAO) [5], a set of linear transfer functions, is used for modelling the linear wave-excitation forces.

The modelling approach presented in this paper is a more general extension of the formulation of [7], where the coupled dynamics of the vessel and the harvester are formulated by using the Newton-Euler equations with eliminated constraints (NEEC), as in Shabana [8], and O'Cathain [9]. The harvester, the specific design of which is currently confidential, is assumed to be a vertical circular cylinder, and the hydrodynamic parameters are computed in WAMIT [10], for both the vessel and the harvester device, and processed using the Matlab/Simulink MSS toolbox [4].

\section{A. Modelling assumptions}

The system dynamics are formulated under the assumption that the $b_{2}$-frame dynamics, between the real motor speed $v_{m}^{b_{2}} \equiv \dot{z}_{m}^{b_{2}}$ and the desired motor speed $v_{d}^{b_{2}}$, is given by the following first order LTI system, after Messineo et al [2] and
Sagatun et al [3]:

$$
\dot{v}_{m}^{b_{2}}=[-\lambda] v_{m}^{b_{2}}+[\lambda] v_{d}^{b_{2}},
$$

where $\frac{1}{\lambda}$ is the time constant of the closed loop speed servo system. The servomotor has an incremental encoder, which measures the drum rotational speed, and the measurement noise is assumed to be white:

$$
W_{e}(t)=\aleph\left\{0, V A R\left(W_{e}\right)\right\} .
$$

The vessel is equipped with a motion reference unit (MRU 5) [11], an accelerometer-based position, velocity and acceleration estimation device, which uses an ad-hoc Kalman filter [12], and provides the measurement [11] of the vessel roll, pitch and yaw angles, corresponding angular rate vectors fixed to the vehicle frame, in addition to the relative (dynamic) heave, surge and sway positions. The MRU 5 sensor specifications express the measurement noise in terms of root-meansquare-error (RMSE). The MRU measurement noise is also modelled as white noise:

$$
W_{M R U}(t)=\left[\begin{array}{lll}
W_{M R U_{1}} & \ldots & W_{M R U_{9}}
\end{array}\right]^{T},
$$

where:

$$
W_{M R U_{i}}(t)=\aleph\left\{0, \sigma_{M R U_{i}}^{2}\right\} .
$$

The harvester device is equipped with a sonar device, which senses the instantaneous seabed profile. To simulate the real sonar measurement, we assume that the sonar has a coneshaped beam dispersion surface, with a dispersion angle $\alpha_{s}$, and an additional (white) measurement noise, $W_{s}(t)$ [13]. In this initial study, we assume the stiffness fo the cable connecting vessel and harvester to be infinitely stiff.

\section{B. The complete system dynamics}

Expanding the formulation of Fossen and Perez [5], a 2body unconstrained 12 DOF motion model can be obtained, as in Gallieri [13]. Then, using the NEEC approach, the model presented here will have 9 DOF. To apply the NEEC, we choose the following $9 \times 1$ independent velocity vector (generalized coordinates):

$$
s=\left[\begin{array}{lllllllll}
u_{1} & v_{1} & w_{1} & p_{1} & q_{1} & r_{1} & p_{2} & q_{2} & \dot{z}_{m}
\end{array}\right]^{T},
$$

defined in Figure 2, except for the harvester roll and pitch rate, $p_{2}$ and $q_{2}$. The partial velocity matrix, $P \in \mathbb{R}^{9 \times 12}$, given by:

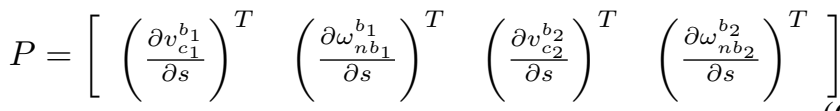

is defined. The generalized velocities, $\nu$, and accelerations $\dot{\nu}$, are given by:

$$
\nu=P^{T} s \quad \dot{\nu}=P^{T} \dot{s}+\dot{P}^{T} s
$$

Defining the vector $q$, such that $\dot{q}=s$, where $s$ is given by eq.(5), we can define the following state, input and output 
vectors, respectively, as:

$$
\underline{\mathbf{x}}=\left[\begin{array}{c}
q \\
s \\
\mu \\
\eta
\end{array}\right], \quad U_{i n}=\left[\begin{array}{c}
\left(\tilde{\tau}_{c}+\tilde{\tau}_{p}\right) \\
U \\
v_{d}
\end{array}\right], \quad \underline{\mathbf{y}}=\left[\begin{array}{c}
\tilde{s} \\
\tilde{\eta}
\end{array}\right] \text {, }
$$

where:

$$
\tilde{s}=\left[\begin{array}{lllllll}
u_{1} & v_{1} & w_{1} & p_{1} & q_{1} & r_{1} & w_{2}
\end{array}\right]^{T},
$$

and $U_{i n} \in \mathbb{R}^{10 \times 1}$, while the vectors, $\tilde{\tau}_{c}$ and $\tilde{\tau}_{p}$, provide the generalized propeller and ship motion control forces in the $b$-frames, as given in [5] and [6], and where $v_{d}$, the desired winch motor speed, is the altitude control (manipulated) signal.

The seaweed harvester dynamics are given by the following non-linear stochastic state space system:

$$
\begin{gathered}
\underline{\dot{\mathrm{x}}}=A(\underline{\mathrm{x}}) \underline{\mathrm{x}}+B(\underline{\mathrm{x}}) U_{i n}+W_{x} \\
\mathrm{y}=C(\underline{\mathrm{x}}) \underline{\mathrm{x}}+W_{y},
\end{gathered}
$$

where the input matrix, $B(\underline{\mathrm{x}}) \in \mathbb{R}^{84 \times 10}$, is given by:

$$
B(\underline{\mathrm{x}})=\left[\begin{array}{llll}
0 & B_{2}^{T}(\underline{\mathrm{x}}) & 0 & B_{4}^{T}
\end{array}\right]^{T}
$$

and $B_{2} \in \mathbb{R}^{9 \times 10}, B_{4} \in \mathbb{R}^{11 \times 10}$, are given by:

$$
B_{2}(\underline{\mathrm{x}})=\left[\begin{array}{ccc}
\tilde{B} & 0 & 0 \\
0 & 0 & \lambda
\end{array}\right], \quad B_{4}=\left[\begin{array}{lll}
0 & \vec{v}_{h}^{n} & 0
\end{array}\right] .
$$

where $\tilde{B}$ is the upper left $(8 \times 8)$ submatrix of $\left(M^{g}\right)^{-1}$, defined in eq.(16). In eq.(12) is assumed that the motor speed input, $v_{d}$, only affects the motor velocity state, $w_{2}$. The state transfer matrix, $A(\underline{\mathrm{x}}) \in \mathbb{R}^{84 \times 84}$, in eq.(10), is given by:

$$
A(\underline{\mathrm{x}})=\left[\begin{array}{cccc}
0 & I & 0 & 0 \\
A_{21}(\underline{\mathrm{x}}) & A_{22}(\underline{\mathrm{x}}) & A_{23}(\underline{\mathrm{x}}) & 0 \\
0 & B_{r}^{g} & A_{r}^{b} & 0 \\
0 & A_{42}(\underline{\mathrm{x}}) & 0 & 0
\end{array}\right]
$$

where $A_{21}(\underline{\mathrm{x}}) \in \mathbb{R}^{9 \times 9}$ is given by:

$$
A_{21}(\underline{\mathrm{x}})=\left[\begin{array}{c}
\widetilde{A}_{21} \\
0
\end{array}\right]
$$

where the matrix $\widetilde{A}_{21}$ contains the first 8 rows of:

$$
A_{21}^{\star}=-\left(M^{g}\right)^{-1} G^{g}
$$

where $G^{g} \in \mathbb{R}^{9 \times 9}$ is the restoring force matrix [5], [14], in the NEEC space [9], where [9]:

$$
M^{g}=P M_{R B}^{b} P^{T} .
$$

is the generalized mass matrix, of the 9 DOF constrained system, in which, $M_{R B}^{b}=\left(J_{b}^{h}\right)^{T} M_{R B}^{h} J_{b}^{h}$ is the rigid body mass matrix in the $b_{1}$ and $b_{2}$-frame, and [9]:

$$
M_{R B}^{h}=\operatorname{blkDiag}\left\{\left[\begin{array}{ll}
M_{R B_{1}}^{h_{1}} & M_{R B_{2}}^{h_{2}}
\end{array}\right]\right\} \text {. }
$$

is the rigid body mass matrix, in the $h_{1}$ and $h_{2}$-frame. In eq. (13) the term $A_{22}(\underline{x}) \in \mathbb{R}^{9 \times 9}$ :

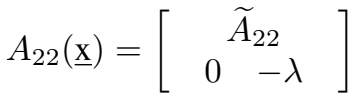

which includes the motor dynamics, where $\widetilde{A}_{22}$ contains the first 8 rows of:

where

$$
A_{22}^{\star}=-\left(M^{g}\right)^{-1}\left(N^{g}(\infty)+D_{r}^{g}+C_{R B}^{g}\right)
$$

with

$$
N^{g}(\infty)=P N^{b}(\infty) P^{T}
$$

$$
N^{b}(\infty)=\left(J_{b}^{h}\right)^{T}\left[B^{h}(\infty)+U A^{h}(\infty) \tilde{L}\right] J_{b}^{h},
$$

and $A^{h}(\infty), B^{h}(\infty)$ are the infinite frequency added mass and damping matrices, given in [5], as $\tilde{L}$, while $J_{b}^{h}$ is given in [13], and where:

$$
C_{R B}^{g}=P M_{R B}^{b} \dot{P}^{T}+P C_{R B}^{b} P^{T} .
$$

provides the rigid body centripetal and Coriolis matrix, where:

$$
C_{R B}^{b}=\left[\begin{array}{cc}
U M_{R B_{1}}^{b_{1}} L & 0 \\
0 & C_{R B_{2}}^{b_{2}}
\end{array}\right]
$$

is the centripetal and Coriolis matrix, in the $b$-frames, and

$$
C_{R B_{2}}^{b_{2}}=\left[\begin{array}{cc}
m_{2} S\left(\omega_{n b_{2}}^{b_{2}}\right) & -m_{2} S\left(\omega_{n b_{2}}^{b_{2}}\right) S\left(r_{c_{2}}^{b_{2}}\right) \\
m_{2} S\left(r_{c_{2}}^{b_{2}}\right) S\left(\omega_{n b_{2}}^{b_{2}}\right) & -S\left(I_{2}^{b_{2}} \omega_{n b_{2}}^{b_{2}}\right)
\end{array}\right],
$$

is the centripetal and coriolis matrix, for a single rigid body, as given in [5], [6], and $L$ is given in [5].

Returning to the terms in eq.(13), $A_{23}(\underline{\mathrm{x}}) \in \mathbb{R}^{9 \times 11 \tilde{n}}$, is given by:

$$
A_{23}(\underline{\mathrm{x}})=\left[\begin{array}{c}
\widetilde{A}_{23} \\
0
\end{array}\right]
$$

where $\tilde{n}$ is the order of the state space approximation of the convolution integral of the Cummins equation [15], [16], and the matrix $\widetilde{A}_{23}$ contains the first 8 rows of:

$$
A_{23}^{\star}=-\left(M^{g}\right)^{-1} C_{r}^{g},
$$

where $C_{r}^{g}$ is defined in eq.(32).

Finally, $A_{42}(\underline{\mathrm{x}}) \in \mathbb{R}^{11 \times 9}$, in eq.(13), is given by:

$$
A_{42}(\underline{\mathrm{x}})=\widetilde{J}_{b}^{n} P^{T},
$$

where $\widetilde{J}_{b}^{n}$ contains the $11 \times 11$ upper matrix of $J_{b}^{n}$, given in [13], and $P$ is given by eq.(6).

The state disturbance vector, $W_{x} \in \mathbb{R}^{84 \times 1}$, appearing in (10) and due to the first and second order wave forces [14], is given by:

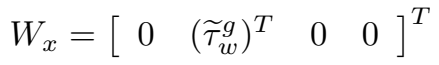


where $\widetilde{\tau}_{w}^{g}$ contains the first 8 elements of the first and second order wave induced force vector, $\tau_{w}^{g}$ [5], which is computed using the RAO approach as in [5] and [14].

The output disturbance vector, $W_{y} \in \mathbb{R}^{14 \times 1}$, in (10), is a stochastic process vector, given as:

$$
W_{y}(t)=\aleph\left\{0, V A R\left(W_{y}\right)\right\},
$$

and depends on the sensor measurement noise, $W_{M R U}, W_{s}$, and $W_{e}(t)$. The output matrix, $C(\underline{\mathrm{x}}) \in \mathbb{R}^{14 \times 84}$, in (10), is given by:

$$
C(\underline{\mathrm{x}})=\left[\begin{array}{cccc}
0 & C_{s} & 0 & 0 \\
0 & 0 & 0 & C_{\eta}(\underline{\mathrm{x}})
\end{array}\right]
$$

where $C_{s}$ and $C_{\eta}(\underline{\mathrm{x}})$, are given in Gallieri [13].

The following provides the fluid memory state space approximation of Cummins' equation [15], computed for both the vessel and the harvester device, using the approach in [16].

$$
\dot{\mu}=A_{r}^{b} \mu+B_{r}^{g} s \quad, \quad \tilde{\mu}^{g}=C_{r}^{g} \mu+D_{r}^{g} s
$$

where:

$$
B_{r}^{g}=B_{r}^{b} P^{T}, C_{r}^{g}=P C_{r}^{b}, D_{r}^{g}=P D_{r}^{b} P^{T},
$$

and $A_{r}^{b}, B_{r}^{b}, C_{r}^{b}$ and $D_{r}^{b}$ are computed with the approach in [16].

\section{Control Design}

For the altitude control of the harvesting device, two control strategies have been implemented and compared. The first strategy, which uses a gain scheduled PID controller [13], is shown in Section IV-A.

The second strategy, shown in Figure 3, includes a feedforward controller to counteract the vessel-induced altitude motion of the cutter, and an altitude feedback controller, to follow the seabed profile. The approach combines a model based feed-forward, with a gain scheduled proportional feedback controller, and is shown in Section IV-B.

The feed-forward controller tries to linearize the harvester heave motion, and simplifies the altitude control problem. The feed-forward scheme, shown in Figure 3, uses the Seatex MRU and the seaweed harvester kinematics to compute the nonlinear motion of the harvester, induced by the vessel, which is the input for the feed forward (FF) controller. Assuming that the motor dynamics are linear, the FF control law will be a LTI discrete time transfer function. The wave induced disturbances, $d(k)$, acting on the harvester altitude, are a function of the system state $\underline{\mathbf{x}}$. To compute the current value of $d(k)$, an estimator is needed, as shown in Section IV-B1. The feedback scheme, shown in Figure 3, uses the sonar device to sense the distance between the harvester and the seabed. A Nomoto PID vessel heading controller [5], included in the MSS toolbox, is used for course keeping.
Vessel Feed Forward

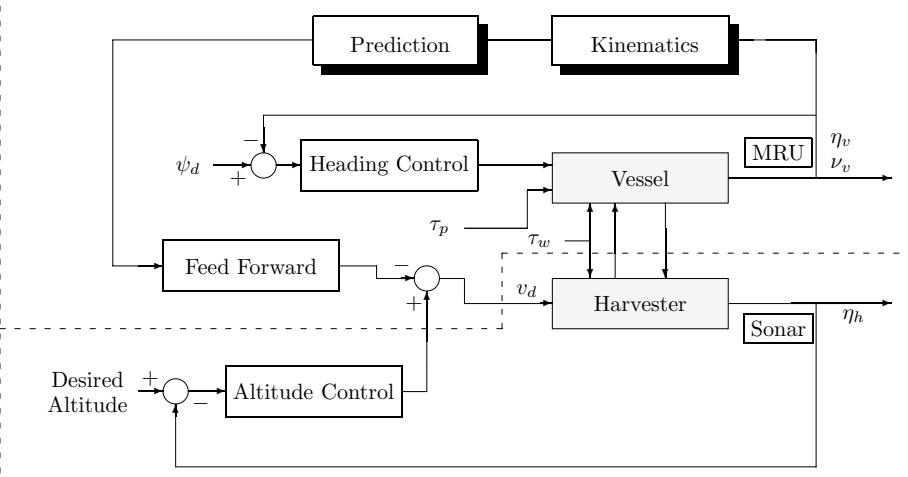

Altitude Feedback

Fig. 3. The altitude control scheme

\section{A. Gain scheduled PID altitude controller}

Initially, a stand alone discrete time PID controller, with adapting coefficients, is implemented. The feedback law is given by the recursive velocity PID algorithm [17]:

$$
\begin{array}{cc}
v_{d}(k)= & v_{d}(k-1)+K_{p}(e(k)-e(k-1))+K_{i} T_{s} e(k) \\
& +K_{d} / T_{s}(e(k)-2 e(k-1)-e(k-2)) \\
\text { where } & e(k)=y_{d}-y(k),
\end{array}
$$

where $y(k)$ is the altitude measure, $y_{d}$ is the altitude setpoint, $T_{s}=0.05 s$ is the sample time. The control law of eq.(33) is adapted, according to the error magnitude, using the following $K_{p}=\left\{\begin{array}{cc}K_{p}^{M A X}, & |e(k)| \geq e^{\star} \\ \left(K_{p}^{M A X}-K_{p}^{M I N}\right) \frac{|e(k)|}{e^{\star}}+K_{p}^{M I N}, & |e(k)|<e^{\star}\end{array}\right.$

$K_{i}=\left\{\begin{array}{cl}K_{i}^{M A X}, & |e(k)| \geq e^{\star} \\ \left(K_{i}^{M A X}-K_{i}^{M I N}\right) \frac{|e(k)|}{e^{\star}}+K_{i}^{M I N}, & |e(k)|<e^{\star}\end{array}\right.$

$K_{d}=\left\{\begin{array}{cc}K_{d}^{M I N}, & |e(k)| \geq e^{\star} \\ \left(K_{d}^{M I N}-K_{d}^{M A X}\right) \frac{|e(k)|}{e^{\star}}+K_{d}^{M A X}, & |e(k)|<e^{\star}\end{array}\right.$

where $e^{\star}=0.1 \mathrm{~m}, K_{p}^{M A X}=10.264, K_{p}^{M I N}=8$, $K_{i}^{M A X}=0.5906, K_{i}^{M I N}=0.1, K_{d}^{M A X}=0.002, K_{d}^{M I N}=$ 0.0001 .

\section{B. Combined feedback and feed-forward control}

1) The feed-forward vessel motion compensator: The harvester motion satisfies the following kinematic relation [13]:

$$
\begin{aligned}
\dot{z}_{2} & =R_{2}^{n} w_{2} \\
& =\operatorname{Row}_{3}\left\{R_{1}^{n}\right\}\left[\begin{array}{c}
u_{1} \\
v_{1} \\
w_{1}
\end{array}\right]+\operatorname{Row}_{3}\left\{R_{1}^{n} S^{T}\left(r_{o_{2}}^{b_{1}}\right)\right\}\left[\begin{array}{c}
p_{1} \\
q_{1} \\
r_{1}
\end{array}\right] \\
& +\operatorname{Row}_{3}\left\{R_{2}^{n}\right\}\left[\begin{array}{c}
l_{c_{2}} q_{2} \\
-l_{c_{2}} p_{2} \\
v_{m}
\end{array}\right]
\end{aligned}
$$


Assuming that the position, velocity and acceleration of the vessel are measurable, taking the small angle approximation for the harvester motion, and substituting the results of eq.(1), we obtain the following model:

$$
\begin{gathered}
\dot{v}_{m}(t)=-\lambda v_{m}(t)+\lambda v_{d} \\
\dot{z}_{2}(t)=v_{m}(t)+d(t)+d_{w}(t),
\end{gathered}
$$

where

$$
\begin{aligned}
d(t)= & -s \theta_{1} u_{1}+c \theta_{1} s \phi_{1} v_{1}+c \theta_{1} c \phi_{1} w_{1}-\bar{z}_{o_{2}} c \theta_{1} s \phi_{1} p_{1} \\
& -\left[\bar{z}_{O_{2}} s \theta_{1}+\bar{x}_{o_{2}} c \theta_{1} c \phi_{1}\right] q_{1}+\left[\bar{x}_{O_{2}} c \theta_{1} s \phi_{1}\right] r_{1}
\end{aligned}
$$

is the vessel induced heave velocity disturbance, which is a function of the vessel attitude and speed, while

$$
d_{w}(t)=\aleph\left\{0, V A R\left(d_{w}\right)\right\},
$$

is a Gaussian white noise disturbance, including the model uncertainties and measurement noise. From eq.(39), we have:

$$
\begin{gathered}
v_{m}(s)=\frac{\lambda}{s+\lambda} v_{d}, \\
z_{2}(s)=\frac{1}{s}\left(v_{m}(s)+d(s)+d_{w}(s)\right) .
\end{gathered}
$$

Choosing the following feed-forward control law:

$$
v_{d}(s)=-\gamma \frac{s+\lambda}{\lambda(s+\gamma)} d(s)
$$

the system dynamic becomes:

$$
z_{2}(s)=\frac{1}{s+\gamma} d(s)+\frac{1}{s} d_{w}(s)
$$

A discrete-time equivalent, using Tustin's method [17], for eq.(43) is:

$$
z_{2}(k)=\frac{T_{s}}{2} \frac{z+1}{z-1}\left(v_{m}(k)+d(k)+d_{w}(k)\right) .
$$

and the control action, $v_{d}(k)$, is given by:

$$
v_{d}(k)=k \frac{z-\alpha_{1}}{z-\beta_{1}} d(k),
$$

where $k, \alpha_{1}$ and $\beta_{1}$ are given in [13]. To apply the control action, given by eq.(47), we need an estimate, $\hat{d}(k)$, of the vessel induced disturbance, $d(k)$. Given $\hat{d}(k)$, and defining an estimation error as $\hat{e}(k)=d(k)-\hat{d}(k)$, the closed loop dynamics become:

$$
z_{2}(k)=\frac{T_{s}(z+1)}{z-\beta_{1}} \hat{d}(k)+\frac{T_{s}(z+1)}{2(z-1)}\left(\hat{e}(k)+d_{w}(k)\right) .
$$

The quality of the control is therefore a function of the accuracy of $\hat{d}(k)$. Given the high complexity of the 9 DOF model, for the aim of the feasibility study, several black-box estimation strategies for the wave induced disturbance, $d(k)$, have been evaluated:

- Previous sample holding (PSH), where:

$$
\hat{d}(k)=d(k-1)=z^{-1} d(k),
$$

providing an error given by [13]:

$$
\hat{e}(t)=T_{s} d \dot{(t)},
$$

- Recursive least squares autoregressive (AR) model identification (RAR) of $d(k)$, using a forgetting factor [13], [18], [19],

- Multiple switching AR identification (MSAR), where $d(k)$ is estimated using a set of AR models with fixed parameters, along with an adaptive recursive least squares (RLS) AR model, to ensure asymptotic stability, and a reconfigurable RLS AR model. At every iteration, a logical supervisor uses the estimation error of every model to select the next candidate, to perform the estimation [20].

- Periodical least square AR identification (PLSAR), which periodically uses the offline LS algorithm, over a certain number of samples, to identify an AR model, used to predict the next sample of $d(k)$.

The model order can be chosen with an appropriate data analysis and cross validation, as shown in [13], [18]. To date, the best control results have been achieved by using the PLSAR approach, as shown in Gallieri [13].

2) The gain scheduled feedback loop: Combining the predictive heave feed-forward, shown in Section IV-B1, with a gain scheduling $\mathrm{P}$ controller, we obtain the following control law:

$$
v_{d}(k)=\tilde{v}_{d}(k)+v_{d}^{\star}(k),
$$

where $\tilde{v}_{d}(k)$ is given by the feed forward controller of eq.(47), using the PLSAR disturbance estimation [13] , and

$$
v_{d}^{\star}(k)=K_{p} e(k),
$$

where $K_{p}$ is adapted with the rule of, eq.(35), with $e^{\star}=$ $0.1 \mathrm{~m}$, and $K_{p}^{M A X}=3, K_{p}^{M I N}=1.5$.

\section{Control Results}

The simulated system is subject to variations in free surface elevation and seabed profile as shown in Fig.4. The surface variations come from a JONSWAP [21] model with a significant wave height of $1.5 \mathrm{~m}$, a directional spreading factor of 4 [6] and the mean wave direction, relative to the initial vessel heading, is $\pi$ rads/s. The seabed variations constitute both abrupt and smooth changes, with a variance of $2.15 \mathrm{~m}$ around the mean seabed height. These initial control results neglect measurement noise. The control performances of the gain scheduling PID controller, are shown in Figure 5 , with a setpoint of $0.8 \mathrm{~m}$, while the performance of a feedforward controller with a gain scheduled proportional feedback controller is shown in Fig.6.

\section{CONCLUSION}

In this paper, the feasible altitude control of a seaweed harvester, with respect to an unknown seabed profile, has been proven. The combined adaptive feed forward and feedback control strategy offers a balance between low complexity, moderate sensor requirements, and control accuracy. From the 


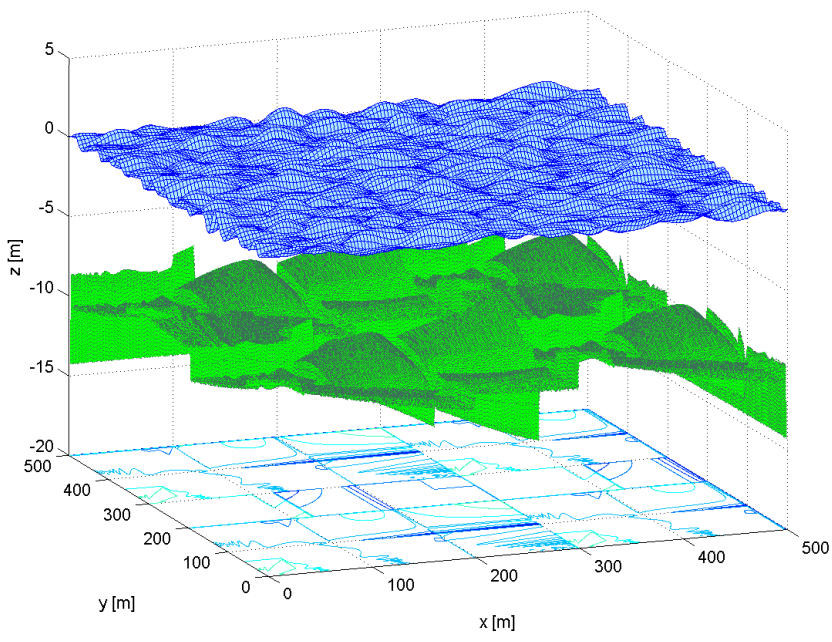

Fig. 4. Variations in seabed profile and free surface elevation

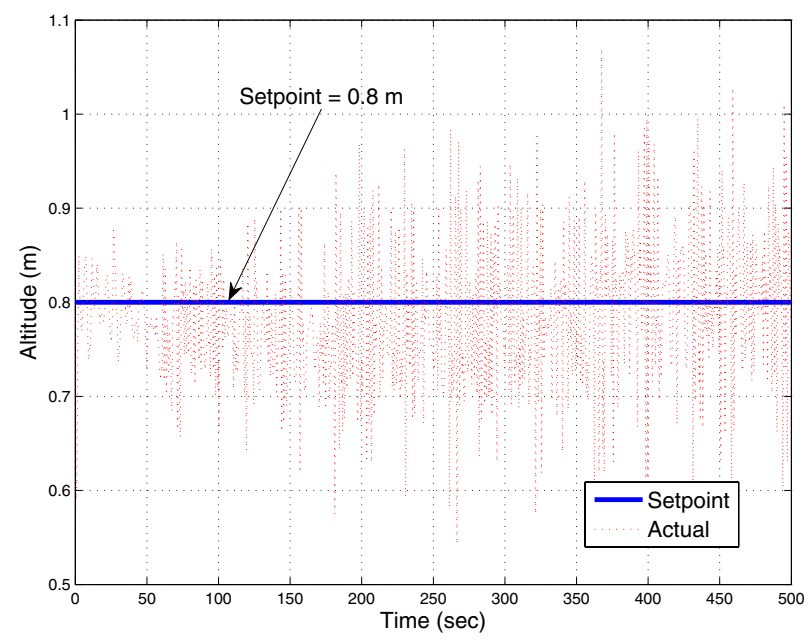

Fig. 5. Altitude response, using the gain scheduled PID altitude control

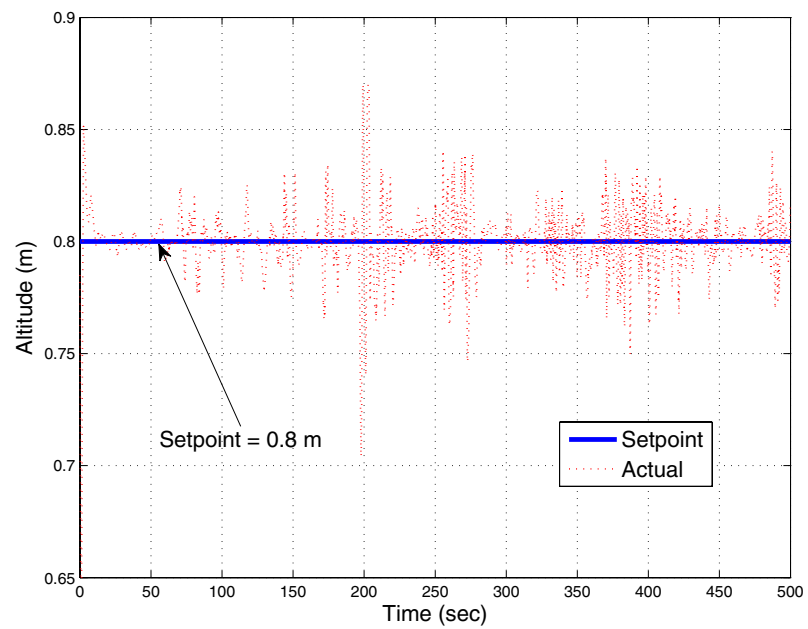

Fig. 6. Altitude response, using the combined proportional and feed-forward control simulation results, in the presence of ideal sensors, the altitude control error is less than $\pm 5 \mathrm{~cm}$.

While this preliminary study offers encouragement, a number of areas need to be addressed, including the effects of sensor noise and modelling/linearisation errors, to which feedforward control strategies can be sensitive. Finite stiffness of the harvester cable should also be considered by including an extra degree of freedom in the model, resulting in a modification of the restoring force matrix [5].

\section{REFERENCES}

[1] P. Falat, L. Brzozowska, and K. Brzozowski, "Application of neural network to control the load motion of an offshore crane," in IEEE Workshop on Intell. Data Acq. and Advanced Comp. Systems: Tech. and Apps., Sofia, Bulgaria, 5-7 September 2005.

[2] S. Messineo, F. Celani, and O. Egeland, "Crane feedback control in offshore moonpool operations," Control Eng. Practice, vol. 16, p. 356:364, 2008.

[3] S. Sagatun, T. Johansen, T. Fossen, and F. Nielsen, "Wave synchronizing crane control during water entry in offshore moonpool operations," IEEE J. Oceanic Eng., vol. 28, p. 720:728, 2003.

[4] T. Perez, O. Smogeli, T. Fossen, and A. Sorensen, "An overview of the marine system simulator MSS: A Simulink toolbox for marine control systems," Modelling, Ident. and Control, vol. 27, no. 4, p. 259:275, 2006.

[5] T. Perez, Ship motion control. Course keeping and roll stabilization using rudder and fins. Springer, 2005.

[6] T. Fossen, Marine Control Systems: Guidance, Navigation and Control of Ships, Rigs and Underwater Vehicles, M. Cybernetics, Ed. Trondheim: Marine Cybernetics, 2002.

[7] M. Gallieri and J. Ringwood, "Optimal harvesting patterns for a seaweed harvester," in Proc. 20th Irish Sig. and Syst. Conf., Dublin, Ireland, June 2009.

[8] A. A. Shabana, Dynamics of Multibody Systems, 3rd ed. Camb., 2004

[9] M. OCathin, B. Leira, J. Ringwood, and J.-C. Gilloteaux, "Modelling of multibody marine systems with application to wave-energy devices," Ocean Eng., vol. 35, p. 1381:1387, 2008.

[10] WAMIT User Manual, Version 6.4, 6.4PC, 6.4S, 6.4S-PC, Wamit inc., available online on www.wamit.com.

[11] Seatex, Seatex MRU User's Manual, 11th ed., 2006.

[12] A. H. Jazwinski, Stochastic process and filtrering theory, A. Press, Ed. Academic Press, New York, 1970.

[13] M. Gallieri, "Feasible altitude control of a seaweed harvester," Dept. of Electronic Eng., Nat. Univ. of Ireland, Maynooth., Tech. Rep., 2009.

[14] J. M. Journee and W. W. Massie, Offshore Hydromechanics - $1^{\text {st }}$ Edition. Delft Univ. of Technology., 2001.

[15] W. Cummins, "The impulse responce function and ship motion," David Taylor Model Basin-DTNSRDC, Tech. Rep. 1661, 1962.

[16] E. Kristiansen and O. Egeland, "Frequency dependent added mass in models for controller design for wave motion ship damping," in 6th IFAC Conf. on Manoeuvring and Control of Marine Craft (MCMC), Girona, Spain, 2003.

[17] R. Isermann, Digital Control Systems. New York: Springer, 1981.

[18] L. Ljung, Identification: Theory for the user, Prentice-Hall, Ed. Prentice-Hall, 1987.

[19] R. Kalman, P. Falb, and M. Arbib, Topics in Mathematical Systems Theory, M. Hill, Ed. McGraw Hill Book Company, 1969, vol. 8.

[20] K. Narendra, J. Balakrishnan, and M. K. Ciliz, "Adaptation and learning using adaptive models, switching and tuning," IEEE Control Syst. Mag., vol. 15 , p. $37: 51,1995$

[21] K. Hasselmann and D. Olbers, "Measurements of wind-wave growth and swell decay during the joint north sea wave project (JONSWAP)," Ergnzung zur Deut. Hydrogr. Z., Reihe A, vol. 8, no. 12, p. 1:95, 1973. 